\title{
Matching fields of a long superconducting film
}

\author{
Edson Sardella \\ Departamento de Física, Universidade Estadual Paulista, Caixa Postal 473, 17033-360, Bauru-SP, Brazil
}

M. M. Doria

Instituto de Física, Universidade Federal do Rio de Janeiro, Caixa Postal 68528, 21945-970, Rio de Janeiro-RJ, Brazil

P. R. S. Netto

Departamento de Física, Universidade Estadual Paulista, Caixa Postal 473, 17033-360, Bauru-SP, Brazil

(Received 6 May 1999)

\begin{abstract}
We obtain the vortex configurations, the matching fields, and the magnetization of a superconducting film with a finite cross section. The applied magnetic field is normal to this cross section, and we use the London theory to calculate many of its properties, such as the local magnetic field, the free energy, and the induction for the mixed state. Thus previous similar theoretical works, done for an infinitely long superconducting film, are recovered here, in the special limit of a very long cross section. [S0163-1829(99)00242-8]
\end{abstract}

\section{INTRODUCTION}

The superconducting properties of an infinite type-II film have been studied for more than three decades. As long ago as 1964, it was shown by Abrikosov ${ }^{1}$ that when an infinitely long film is placed in an external magnetic field, parallel to its surfaces, vortices penetrate collectively into the sample in the form of linear chains. He also calculated the lower critical field $H_{c 1}(b)$ as a function of the film thickness $b$ and shown that for small $b$, it becomes increasingly more difficult for a linear chain of vortices to penetrate the film. After the work of Abrikosov, many theoretical ${ }^{1-5}$ and experimental works ${ }^{6-10}$ have been carried out to study the mixed state of a type-II superconducting film. As the external magnetic field exceeds the value of $H_{c 1}(b)$, the linear chains of vortices start to penetrate into the sample, one at the time. This reflects into the magnetization, which exhibits a succession of peaks related to the penetration of each linear chain. The transition between two vortex states, differing by a linear chain, occurs at a well-defined critical magnetic field, known as the matching field.

In this paper we determine the matching fields and the magnetization of a film of finite cross section, namely, with a finite width $a$ and also with a finite thickness $b$. The applied field is always normal to this cross section, so that the third dimension is also taken very long here. For $b$ very large we recover the results obtained by the previous authors in the limit of an infinitely long film. Interestingly there are two kinds of matching fields in this geometry, because vortices penetrate individually here. The first kind corresponds to the critical field for the penetration of an individual vortex. Thus there are many of this critical field, each delimiting the transition from a $N-$ to a $(N+1)-$ vortex state. The second kind is associated to a transition between different chain states, like in the case of an infinitely long film. As vortices enter the finite film, they align themselves and form a linear chain, parallel to the longer side of the film. As the number of vortices increase inside the sample, the linear chain saturates and undergoes a transition, splitting into two new chains. This process continues from two to three chains and so on. The critical fields associated with these transitions correspond to that required to enter a new linear chain in the case of the infinitely long film.

In summary we show here several new properties of the finite film, such as in the formation of the equilibrium vortex state. The source of these new effects lays in the strong and very important border effects that do not exist in case of the infinitely long film. For instance, a vortex state made of parallel chains is substantially affected near the edges of the longer side of the film. In order to allow for any possible configuration, we let the positions of the vortices entirely free and minimize the Gibbs free energy with respect to them using a numerical procedure. To this end, we use the Monte Carlo simulated annealing minimization method. This method allows us to search for the global minimum of the Gibbs free-energy (or at least very close to the global minimum) of the vortex system, which corresponds to the most stable configuration. We also solve here for the matching fields, which requires previous knowledge of the Gibbs free-energy minimum for a fixed number of vortices. Notice that this program is much simpler for an infinite film, because there one can suppose some type of symmetry for the vortex state, and minimization can be reduced to a small set of parameters, such as the lattice spacing and the number of chains. ${ }^{5}$

Our analysis is restricted to the low-magnetic-field regime and to the very strong type-II superconductors, where the vortex cores do not overlap and the distance over which the magnetic field penetrates into the sample is much larger than the size of the cores. Within this regime, the mixed state may be well described by the London theory accompanied by some regularization procedure to cure some divergencies that appear at short length scales. To the best knowledge of the present authors this study has not been done before.

\section{MIXED STATE}

The description of the mixed state of a type-II superconductor involves two fundamental lengths. One is the distance 


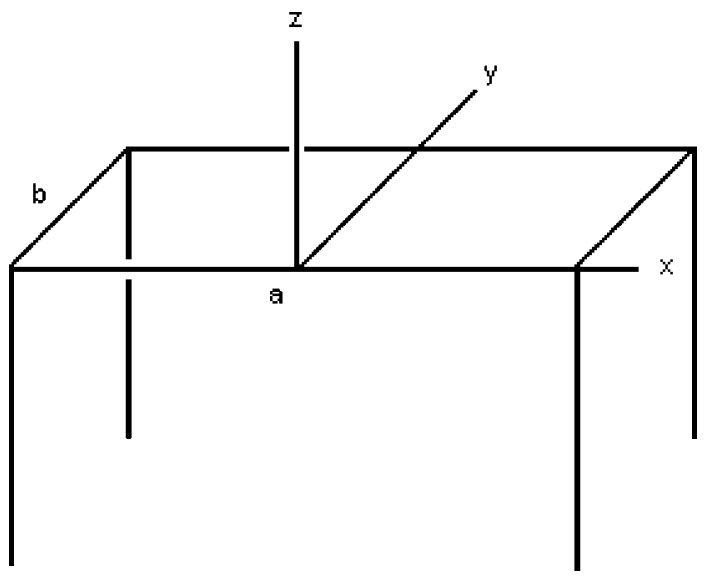

FIG. 1. Cross section of the superconducting film. The external magnetic field is along the $z$ axis and its value is $H$ for $|x|>a / 2$, and $y<0$ and $y>b$.

over which the magnetic field penetrates into the sample, the penetration length $\lambda$. The other one is the coherence length, the size of the vortex core. Our treatment will be limited to the case of a strong type-II superconductor where the Ginzburg-Landau parameter $\kappa=\lambda / \xi \gg 1$. Vortices interact strongly since the distance between them can be much smaller than $\lambda$. Their repulsive interaction forbids overlapping, namely the onset of vortices with multiple vorticity. In this context, the distribution of the local magnetic field may be well described by the London equation. In rectangular coordinates $(x, y)$ the London equation for the local magnetic field $\mathbf{h}=h \mathbf{z}$ is given by

$$
-\lambda^{2} \nabla^{2} h+h=\Phi_{0} \sum_{i} \delta\left(\mathbf{r}-\mathbf{r}_{i}\right),
$$

where $\Phi_{0}$ is the quantum flux and $\mathbf{r}_{i}=\left(x_{i}, y_{i}\right)$ is the position of the $i$ th vortex. We have assumed that the vortices are straight lines. Therefore, a three-dimensional (3D) problem is reduced to a $2 \mathrm{D}$ one.

The geometry of the problem is illustrated in Fig. 1. Let $a$ and $b$ be the sides of the superconducting film. We will solve this equation subject to the following boundary conditions:

$$
\begin{gathered}
h( \pm a / 2, y)=h(x, 0)=h(x, b)=H, \\
\left(\frac{\partial h}{\partial x}\right)_{y=0, b}=\left(\frac{\partial h}{\partial y}\right)_{y= \pm a / 2}=0,
\end{gathered}
$$

where $H$ is the magnitude of the external magnetic field at the interface vacuum superconductor and is pointing along the $z$ direction. The second condition imposes that the supercurrents are confined into the sample.

Other authors have used the image method to solve the London equation (see, for instance, Refs. 3 and 4). We prefer to solve this equation directly, by using the Green's function method. The equation for this function associated with the London equation is given by

$$
-\lambda^{2} \nabla^{2} G+G=\delta\left(x-x^{\prime}\right) \delta\left(y-y^{\prime}\right),
$$

where $G$ must satisfy the following boundary conditions on the film borders

$$
G( \pm a / 2, y)=G(x, 0)=G(x, b)=0 .
$$

We can find an expression for the local magnetic field in terms of the Green's function by multiplying Eq. (1) by $G\left(x, y, x^{\prime}, y^{\prime}\right)$ and Eq. (3) by $h(x, y)$, subtract the results, and then integrate what is left. One obtains

$$
\begin{aligned}
h\left(x^{\prime}, y^{\prime}\right)= & \phi_{0} \sum_{i} G\left(x_{i}, y_{i}, x^{\prime}, y^{\prime}\right) \\
& -H\left[1-\int_{-a / 2}^{a / 2} d x \int_{0}^{b} d y G\left(x, y, x^{\prime}, y^{\prime}\right)\right],
\end{aligned}
$$

where we have made use of the boundary conditions (2) and (4).

Then, the solution for the local magnetic field is transferred to the determination of the Green's function. The approach used to find the solution for this function is sketched in full detail in Ref. 11, except for the fact that there the boundary conditions are taken at infinite. Here we only show the main steps of how to calculate this function. First of all, we expand $G$ in a Fourier series

$$
G\left(x, y, x^{\prime}, y^{\prime}\right)=\frac{2}{b} \sum_{m=1}^{\infty} \sin \left(\frac{m \pi y^{\prime}}{b}\right) \sin \left(\frac{m \pi y}{b}\right) g_{m}\left(x, x^{\prime}\right),
$$

which satisfies the boundary conditions of Eq. (4) at $y$ $=0, b$.

Inserting this equation into Eq. (3) we find for $g_{m}$,

$$
-\lambda^{2} \frac{\partial^{2} g_{m}}{\partial x^{2}}+\alpha_{m}^{2} g_{m}=\delta\left(x-x^{\prime}\right),
$$

where we have used the fact that the sequence

$$
\left\{\sqrt{\frac{2}{b}} \sin \left(\frac{m \pi y}{b}\right) m=1,2,3 \ldots\right\}
$$

is a complete set of orthonormal functions, that is,

$$
\frac{2}{b} \sum_{m=1}^{\infty} \sin \left(\frac{m \pi y^{\prime}}{b}\right) \sin \left(\frac{m \pi y}{b}\right)=\delta\left(y-y^{\prime}\right) .
$$

Here

$$
\alpha_{m}=\left[1+\lambda^{2}\left(\frac{m \pi}{b}\right)^{2}\right]^{1 / 2} .
$$

The function $g_{m}\left(x, x^{\prime}\right)$ must satisfy the same boundary conditions as $G\left(x, y, x^{\prime}, y^{\prime}\right)$, that is, $g_{m}\left( \pm a / 2, x^{\prime}\right)=0$. In addition, the derivative of $g_{m}\left(x, x^{\prime}\right)$ is discontinuous at $x=x^{\prime}$, which results from the $\delta$ function on the right-hand side of Eq. (7).

Under these conditions, the solution for $g_{m}\left(x, x^{\prime}\right)$ is given by

$$
\begin{aligned}
g_{m}\left(x, x^{\prime}\right)= & \frac{1}{2 \lambda \alpha_{m} \sinh \left(\alpha_{m} a / \lambda\right)}\left\{\cosh \left[\alpha_{m}\left(\left|x-x^{\prime}\right|-a\right) / \lambda\right]\right. \\
& \left.-\cosh \left[\alpha_{m}\left(x+x^{\prime}\right) / \lambda\right]\right\} .
\end{aligned}
$$


This completes the solution for the Green's function. To proceed, we still have to find the integral of the Green's function that appears in Eq. (5). This involves a tedious, but straightforward algebra. One has

$$
\begin{aligned}
\int_{-a / 2}^{a / 2} d x \int_{0}^{b} d y G\left(x, y, x^{\prime}, y^{\prime}\right) \\
=\frac{4}{b} \sum_{m=1}^{\infty} \frac{(-1)^{m}}{\alpha_{2 m+1}^{2}} \frac{b}{(2 m+1) \pi} \\
\quad \times \sin \left(\frac{m \pi y^{\prime}}{b}\right)\left[1-\frac{\cosh \left(\alpha_{2 m+1} x^{\prime} / \lambda\right)}{\cosh \left(\alpha_{2 m+1} a / 2 \lambda\right)}\right] .
\end{aligned}
$$

Notice that this vanishes at $x^{\prime}= \pm a / 2$ or $y^{\prime}=0, b$, so that the second term of Eq. (5) is just $H$ as required by the boundary conditions of Eq. (2).

Next we can simplify Eq. (11) by noticing that

$$
\begin{aligned}
\frac{4}{b} \sum_{m=1}^{\infty} \frac{(-1)^{m}}{\alpha_{2 m+1}^{2}} \frac{b}{(2 m+1) \pi} \sin \left(\frac{m \pi y^{\prime}}{b}\right) \\
\quad=\int_{0}^{b} d y g\left(y, y^{\prime}\right)=1-\frac{\cosh \left[\left(y^{\prime}-b / 2\right) / \lambda\right]}{\cosh (b / 2 \lambda)},
\end{aligned}
$$

where

$$
\begin{aligned}
g\left(y, y^{\prime}\right)= & \frac{2}{b} \sum_{m=1}^{\infty} \frac{1}{\alpha_{m}^{2}} \sin \left(\frac{m \pi y^{\prime}}{b}\right) \sin \left(\frac{m \pi y}{b}\right) \\
= & \frac{1}{2 \lambda \sinh (b / \lambda)}\left\{\cosh \left[\left(\left|y-y^{\prime}\right|-b\right) / \lambda\right]\right. \\
& \left.-\cosh \left[\left(y+y^{\prime}\right) / \lambda\right]\right\} .
\end{aligned}
$$

By combining Eqs. (5), (11), and (12) we finally obtain for the local magnetic field

$$
\begin{aligned}
h(x, y)= & \Phi_{0} \sum_{i} G\left(x, y, x_{i}, y_{i}\right)+H\left\{\frac{\cosh [(y-b / 2) / \lambda]}{\cosh (b / 2 \lambda)}\right. \\
& +\frac{4}{b} \sum_{m=0}^{\infty} \frac{(-1)^{m}}{\alpha_{2 m+1}^{2}} \frac{b}{(2 m+1) \pi} \\
& \left.\times \sin \left[\frac{(2 m+1) \pi y}{b}\right] \frac{\cosh \left(\alpha_{2 m+1} x / \lambda\right)}{\cosh \left(\alpha_{2 m+1} a / 2 \lambda\right)}\right\},
\end{aligned}
$$

where we have made use of the following symmetry property of the Green's function, $G\left(x, y, x^{\prime}, y^{\prime}\right)=G\left(x^{\prime}, y^{\prime}, x, y\right)$. We can see that the local magnetic field is composed essentially of three contributions. The last two terms, proportional to $H$ in Eq. (14), represent the penetration of the external magnetic field near the surface when the vortices are still absent. Once the vortices start penetrating the sample they will be trapped by the shielding currents associated with the last two terms of Eq. (14). The Green's function in Eq. (14) contains two terms [see Eqs. (6) and (10)]. The first one, which depends on $\left|x-x_{i}\right|$, represents the local magnetic field of the vortices located inside the sample, and the second one, which depends on $x+x_{i}$, is the local magnetic field of the image vortices located outside the sample.

The free energy per unit volume of the superconducting film is given by

$$
\begin{aligned}
\mathcal{F}= & \frac{1}{8 \pi A} \int d^{2} r\left\{h^{2}+\lambda^{2}\left[\left(\frac{\partial h}{\partial x}\right)^{2}+\left(\frac{\partial h}{\partial y}\right)^{2}\right]\right\} \\
= & \frac{\Phi_{0}}{8 \pi A} \sum_{i} h\left(x_{i}, y_{i}\right)+\frac{H \lambda^{2}}{8 \pi A}\left\{\int _ { 0 } ^ { b } d y \left[\left(\frac{\partial h}{\partial x}\right)_{x=a / 2}\right.\right. \\
& \left.\left.-\left(\frac{\partial h}{\partial x}\right)_{x=-a / 2}\right]+\int_{-a / 2}^{a / 2} d x\left[\left(\frac{\partial h}{\partial y}\right)_{y=b}-\left(\frac{\partial h}{\partial y}\right)_{y=0}\right]\right\},
\end{aligned}
$$

where $A=a b$.

Now notice that by integrating the kinetic term of the London equation of Eq. (1) we obtain precisely the second term of (15). Then, this equation can be simplified to

$$
\mathcal{F}=\frac{\Phi_{0}}{8 \pi A} \sum_{i} h\left(x_{i}, y_{i}\right)+\frac{H B}{8 \pi}-N \frac{\Phi_{0} H}{8 \pi A},
$$

where $N$ is the number of vortices, $B$ is the spatial average of the local magnetic field. The induction is obtained by integrating Eq. (14) over the cross section of the superconducting film. One has

$$
\begin{aligned}
A B= & N \Phi_{0}-\Phi_{0} \sum_{i} \frac{\cosh \left[\left(y_{i}-b / 2\right) / \lambda\right]}{\cosh (b / 2 \lambda)} \\
& -\frac{4 \Phi_{0}}{\pi} \sum_{i} \sum_{m=0}^{\infty} \frac{(-1)^{m}}{\alpha_{2 m+1}^{2}} \frac{1}{(2 m+1)} \\
& \times \sin \left[\frac{(2 m+1) \pi y_{i}}{b}\right] \frac{\cosh \left(\alpha_{2 m+1} x_{i} / \lambda\right)}{\cosh \left(\alpha_{2 m+1} a / 2 \lambda\right)} \\
& \left.+H A\left\{\frac{\tanh (b / 2 \lambda)}{(b / 2 \lambda)}-\frac{8}{\pi^{2}} \sum_{m=0}^{\infty}\right]^{2} \frac{\tanh \left(\alpha_{2 m+1} a / 2 \lambda\right)}{\left(\alpha_{2 m+1} a / 2 \lambda\right)}\right\} .
\end{aligned}
$$

Upon substituting Eq. (17) into Eq. (16) we are left with

$$
\begin{aligned}
\mathcal{F}= & \frac{\Phi_{0}^{2}}{8 \pi A} \sum_{i, j} G\left(x_{i}, y_{i}, x_{j}, y_{j}\right)+\frac{H^{2}}{8 \pi}\left\{\frac{\tanh (b / 2 \lambda)}{(b / 2 \lambda)}\right. \\
& \left.-\frac{8}{\pi^{2}} \sum_{m=0}^{\infty}\left[\frac{1}{(2 m+1) \alpha_{2 m+1}}\right]^{2} \frac{\tanh \left(\alpha_{2 m+1} a / 2 \lambda\right)}{\left(\alpha_{2 m+1} a / 2 \lambda\right)}\right\} .
\end{aligned}
$$

In order to study the most stable configuration of the vortex lattice, it is more convenient to take the Gibbs free energy. The Gibbs free energy per unit volume is given by $\mathcal{G}$ $=\mathcal{F}-B H / 4 \pi$. From Eqs. (17) and (18), it follows that 


$$
\begin{aligned}
\mathcal{G}= & \frac{\Phi_{0}^{2}}{8 \pi A} \sum_{i, j} G\left(x_{i}, y_{i}, x_{j}, y_{j}\right)+\frac{\Phi_{0} H}{4 \pi A} \sum_{i} \frac{\cosh \left[\left(y_{i}-b / 2\right) / \lambda\right]}{\cosh (b / 2 \lambda)}+\frac{\Phi_{0} H}{\pi A} \\
& \times \sum_{i} \sum_{m=0}^{\infty}(-1)^{m} \alpha_{2 m+1}^{2} \frac{1}{(2 m+1)} \sin \left[\frac{(2 m+1) \pi y_{i}}{b}\right] \frac{\cosh \left(\alpha_{2 m+1} x_{i} / \lambda\right)}{\cosh \left(\alpha_{2 m+1} a / 2 \lambda\right)} \\
& -\frac{H^{2}}{8 \pi}\left\{\frac{\tanh (b / 2 \lambda)}{(b / 2 \lambda)}-\frac{8}{\pi^{2}} \sum_{m=0}^{\infty}\left[\frac{1}{(2 m+1) \alpha_{2 m+1}}\right]^{2} \frac{\tanh \left(\alpha_{2 m+1} a / 2 \lambda\right)}{\left(\alpha_{2 m+1} a / 2 \lambda\right)}\right\}-N \frac{\Phi_{0} H}{4 \pi A} .
\end{aligned}
$$

The last two terms are the energy of the Meissner state as if no vortices were present. The second and third contributions are the potential barrier that pin the vortices inside the sample. The Green's function in the first term gives rise to two contributions. One is the repulsive interaction between the vortices and the other one is the attractive interaction between the vortices and the images that are virtually placed outside the sample.

We shall work in the limit of thin film in which $(\pi \lambda / b)^{2} \gg 1$. Within this limit, the sum in $m$ can be evaluated exactly. We proceed as in Ref. 4. One has,

$$
\begin{aligned}
G\left(x_{i}, y_{i}, x_{j}, y_{j}\right)= & \frac{1}{4 \pi \lambda^{2}}\left\{\ln \left[\frac{\cosh \left[\pi\left|x_{i}-x_{j}\right| / b\right]-\cos \left[\pi\left(y_{i}+y_{j}\right) / b\right]}{\cosh \left[\pi\left|x_{i}-x_{j}\right| / b\right]-\cos \left[\pi\left(y_{i}-y_{j}\right) / b\right]}\right]-\ln \left[\frac{\cosh \left[\pi\left(a-x_{i}-x_{j}\right) / b\right]-\cos \left[\pi\left(y_{i}+y_{j}\right) / b\right]}{\cosh \left[\pi\left(a-x_{i}-x_{j}\right) / b\right]-\cos \left[\pi\left(y_{i}-y_{j}\right) / b\right]}\right]\right. \\
& \left.-\ln \left[\frac{\cosh \left[\pi\left(a+x_{i}+x_{j}\right) / b\right]-\cos \left[\pi\left(y_{i}+y_{j}\right) / b\right]}{\cosh \left[\pi\left(a+x_{i}+x_{j}\right) / b\right]-\cos \left[\pi\left(y_{i}-y_{j}\right) / b\right]}\right]\right\} .
\end{aligned}
$$

The first term in Eq. (20) is the same as the one found in Ref. 4. The other two terms are due to the presence of the lateral border of the film. The London theory is not regular for vortex self-interaction. In fact, one can notice that the contribution $i=j$ to the Gibbs free energy (19) gives rise to a logarithmic divergence. We remediate this divergence by using a sharp cutoff in which $\left|x_{i}-x_{j}\right|$ is replaced by $\xi$ for $i=j$. In the limit $(b / \pi \xi) \gg 1$ we obtain

$$
\begin{aligned}
G\left(x_{i}, y_{i}\right)= & \frac{1}{4 \pi \lambda^{2}}\left\{\ln \left[\frac{(\pi \xi / b)^{2}+4 \sin ^{2}\left(\pi y_{i} / b\right)}{(\pi \xi / b)^{2}}\right]-\ln \left[\frac{\cosh \left[\pi\left(a-2 x_{i}\right) / b\right]-\cos \left(2 \pi y_{i} / b\right)}{\cosh \left[\pi\left(a-2 x_{i}\right) / b\right]-\cos (\pi \xi / b)}\right]\right. \\
& \left.-\ln \left[\frac{\cosh \left[\pi\left(a+2 x_{i}\right) / b\right]-\cos \left(2 \pi y_{i} / b\right)}{\cosh \left[\pi\left(a+2 x_{i}\right) / b\right]-\cos (\pi \xi / b)}\right]\right\}
\end{aligned}
$$

The sum over $m$ in the third term on the right-hand side of Eq. (19) cannot be evaluated exactly in the limit $(\pi \lambda / b)^{2}$ $\gg 1$. So it will be kept as it stands.

In the next section we use Eq. (19) combined with Eqs. (20) and (21) to investigate the vortex state in a finite film. This will be carried out via minimization of the Gibbs free energy with respect to the vortex positions upon using the Monte Carlo simulated annealing minimization method.

\section{RESULTS AND DISCUSSION}

The lower critical field, the magnitude of the external field sufficient to create a vortex inside the sample, may be obtained by neglecting the quadratic term in Eq. (19) and equating the remaining terms to zero. We find

$$
H_{c 1}(a, b)=\frac{\Phi_{0}}{2 \lambda^{2}}\left\{\frac{\frac{1}{2 \pi} \ln \left(\frac{2 b}{\pi \xi}\right)-\ln [\{\cosh (\pi a / b)+1\} /\{\cosh (\pi a / b)-1\}]}{1-[1 / \cosh (b / 2 \lambda)]-4 \sum_{m=0}^{\infty}\left[(-1)^{m} /(2 m+1) \pi \alpha_{2 m+1}^{2}\right]\left[1 / \cosh \left(\alpha_{2 m+1} a / 2 \lambda\right)\right]}\right\},
$$

where we have assumed that a single vortex is at the center of the film. It can be easily seen that for $a \rightarrow \infty$ we obtain the same result as in Ref. 5. A quick inspection of Eq. (22) shows us that as $a$ and/or $b$ decreases, $H_{c 1}(a, b)$ increases. In other words, size effects provoke a delay in the first penetration of flux, assuming the external field increasing with time. These finite-size effects have been observed in superconduct- ors of different geometry. ${ }^{12}$ The determination of the other critical fields will be discussed below.

Now we turn our discussion to the minimization of the Gibbs free energy with respect to the vortex positions. Our investigation will be guided by the Monte Carlo simulated annealing minimization method. We have done this from $N$ $=1$ up to $N=30$. We start from an arbitrary initial configu- 

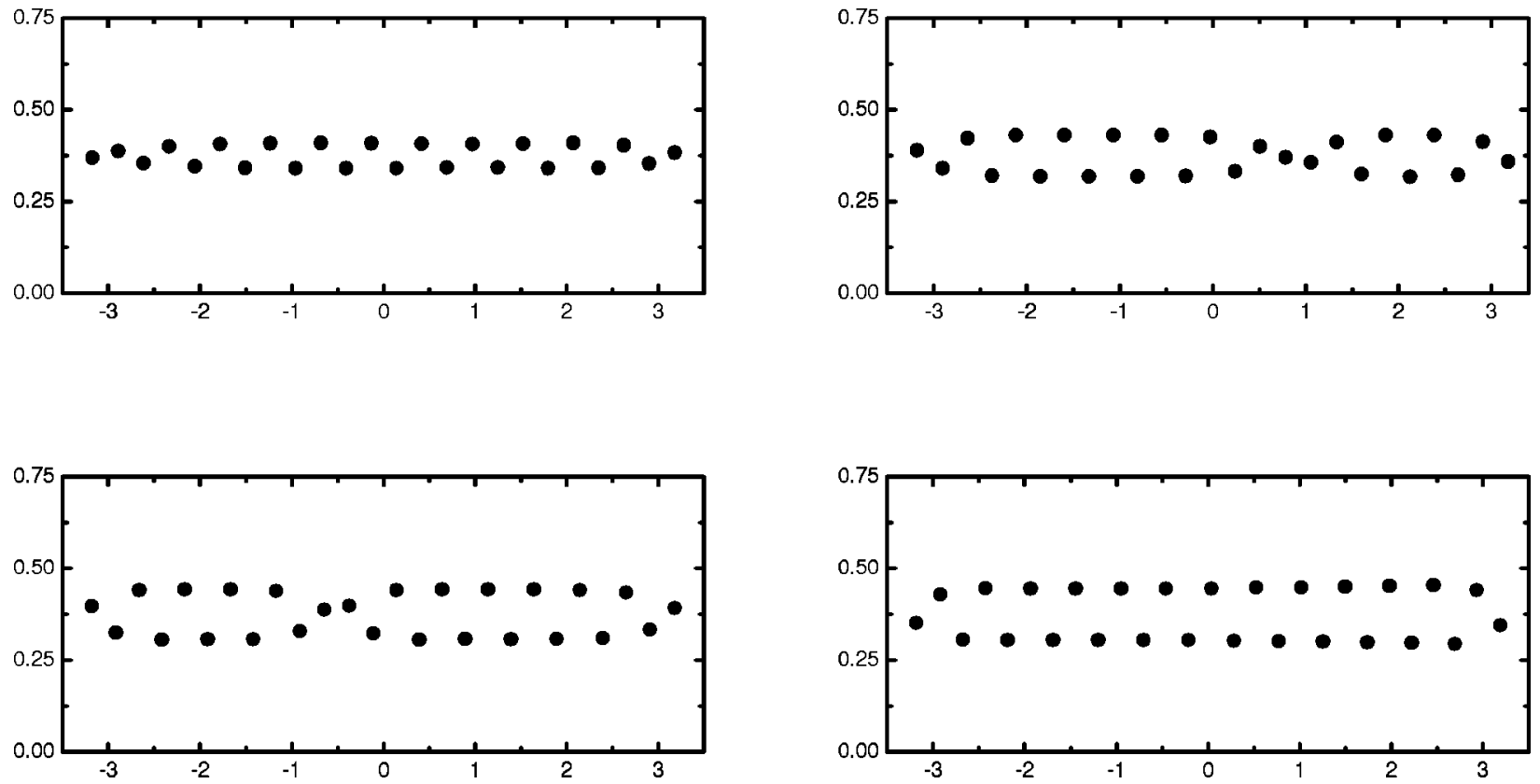

FIG. 2. The double chains for $N=24,25,26,27$, in the clockwise sense. The parameters used are quoted in the text. Notice that the inner vortices make a "zig-zag" as in a triangular lattice. However, at the edges of the film, both chains end up at the same vortex.

ration of the vortex positions at a certain "temperature." By adiabatically lowering this fictitious temperature, simulated annealing will concentrate on the region where it is most likely to succeed.

The parameters we use here are $\kappa=100, a=7 \lambda$, and $b$ $=0.75 \lambda$. We find that, as the penetration of flux is initiated, the vortices will be symmetrically located with respect to the center of the film in form of a linear chain aligned parallel to the $x$ axis (the longer side of the film). As $H$ is increased, at a certain critical value of the external magnetic field, this linear chain breaks into a double chain. At the interior of the film, the vortices arrange in form of a "zig-zag." However, at the edges of the film both linear chains are distorted and joined together (see Fig. 2). For the parameters quoted above, we have found that the change in the symmetry of the lattice occurs at $N=24$. This critical value of $N$ is expected to grow in case the value of the width $a$ of the film becomes larger.

In Fig. 2 we also have shown some other configurations for higher values of $N$. Notice that the vortex state develops some unusual patterns in which the linear chains entangle in the interior of the film. On each step of our numerical calculation, to search the minimum $G$ we lowered the temperature as slowly as possible. However, we cannot assure that the minimum $G$ found corresponds to the global optimum. So, those entangled chain configurations may be metastable solutions.

The penetration of each additional vortex corresponds to a well-defined value of external magnetic field, which we denote by $H_{s N} ; H_{s 1} \equiv H_{c 1}(a, b)$. We find these matching fields by assuming that at the transition from $N$ to $N+1$ vortices, the Gibbs free energy is continuous, that is, $\mathcal{G}_{N}=\mathcal{G}_{N+1}$. Because the vortex positions depend on the external magnetic field, this is a transcendental equation. Full details of how to solve this transcendental equation can be found in Ref. 12. We have determined the sequence of matching fields for the parameters quoted above. We then used these values to calculate the magnetization, which is defined by $M=(B$ $-H) / 4 \pi$.

The magnetization as a function of $H$ is depicted in Fig. 3. At the transitions, the creation of another vortex will force a rearrangement in the chain. This in turn will provoke a discontinuity of the magnetization at $H=H_{s N}$. We can see that the magnetization changes in small steps. So, the magnetization can be used to measure the number of individual vortex penetrations in the sample. Each peak indicates that a new vortex entered the film.

Notice that $-4 \pi M$ has a maximum for $H \geqslant H_{c 1}(a, b)$,

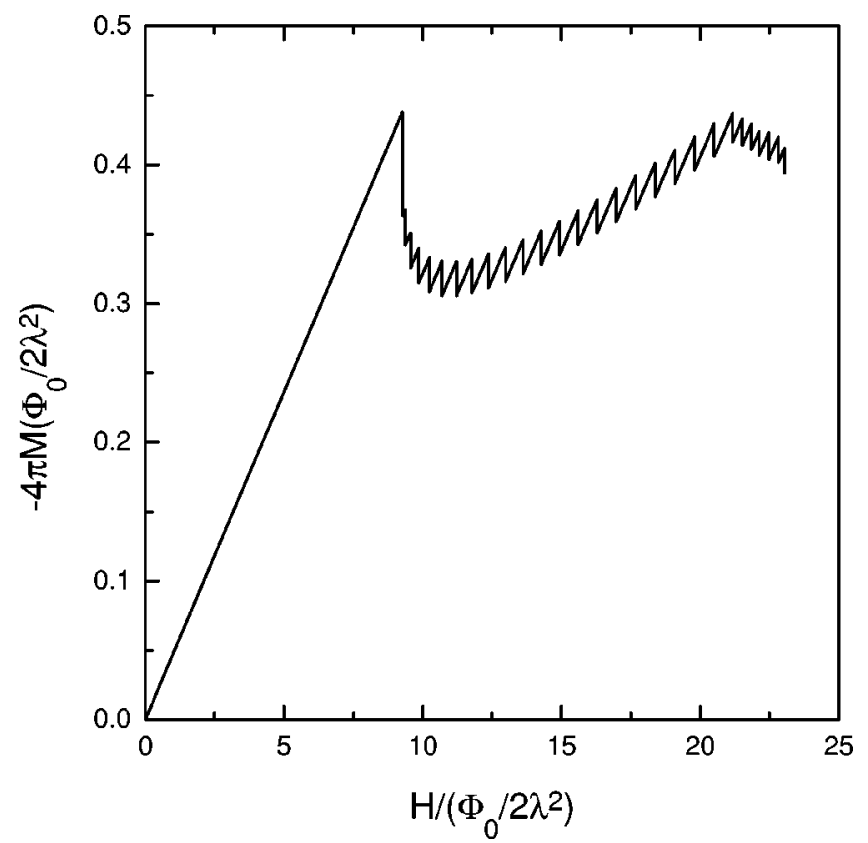

FIG. 3. The magnetization as a function of the external magnetic field $H$. The parameters used are quoted in the text. 


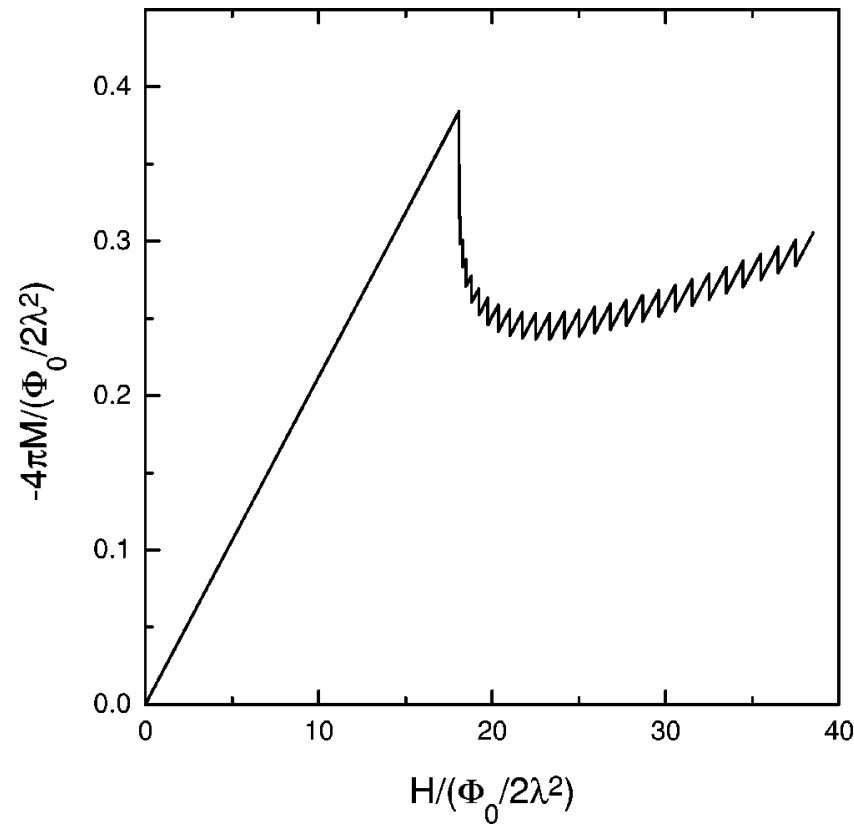

FIG. 4. The magnetization as a function of the external magnetic field $H$. The parameters used are quoted in the text.

signaling a transition from a single to a double chain state, in contrast to superconducting systems with no edges that just show a monotonic behavior. In each phase between $H_{S N}$ and $H_{s, N+1}$, for each value of $H$ we should calculate the corresponding equilibrium configuration of the vortices and use it to evaluate the induction $B$. For the parameters used in Fig. 3, the differences $\Delta H=H_{s, N+1}-H_{s N}$ are not too large.
Then, the vortex positions should not vary significantly with $H$ within each phase. So, to calculate $B$, we held the vortex positions fixed at $H=H_{S N}$. As a consequence, the magnetization varies linearly with $H$ within each phase.

We can also observe that the intensities of the peak of the magnetization at the transition from a single to a double chain lattice, and at $H=H_{c 1}(a, b)$ have approximately the same intensity. In Fig. 4 we repeated the same calculation with $a=7 \lambda$ and $b=0.5 \lambda$. For $N$ up to 30 vortices we did not find any break in the linear chain (see Fig. 4). However, the magnetization curve shows a tendency to reach the second peak of lower magnitude than at $H=H_{c 1}$. On the other hand, for an infinite film, as shown in Ref. 5, the intensity of the magnetization at the phase transitions tends to grow as $H$ increases. We then suspect that this behavior of the magnetization may be a result of size effects.

Finally, we would like to point out that the successive discontinuities of the magnetization discussed here should be experimentally observed. To see this, we note that the order of magnitude of $\Delta H=H_{s, N+1}-H_{s N}$ in Fig. 3 can be as high as $\Phi_{0} / 2 \lambda^{2}$. For zero temperature, $\lambda$ is typically of order $10^{5} \AA$ which gives $\Delta H \sim 10^{-5} \mathrm{~T}$. On the other hand, the jump in the magnetization can achieve the magnitude of order $10^{-7} \mathrm{~T}$. This is well inside the resolution of a magnetometer.

\section{ACKNOWLEDGMENTS}

E.S. thanks the Brazilinan Agencies FAPESP and CNPq for financial support. M.M.D. and P.R.S.N. thank CNPq for financial support.
${ }^{1}$ A. A. Abrikosov, Zh. Éksp. Teor. Fiz. 46, 1464 (1964) [Sov. Phys. JETP 19, 988 (1964)].

${ }^{2}$ V. V. Shmidt, Zh. Éksp. Teor. Fiz. 57, 2095 (1969) [Sov. Phys. JETP 30, 1137 (1970); ibid. 361, 398 (1971) [34, 211 (1972)].

${ }^{3}$ C. Carter, Can. J. Phys. 47, 1447 (1969).

${ }^{4}$ Y. Mawatari and K. Yamafuji, Physica C 228, 336 (1994).

${ }^{5}$ G. Carneiro, Phys. Rev. B 57, 6077 (1998).

${ }^{6}$ S. H. Brongersma, E. Verweij, N. J. Koeman, D. G. de Groot, R. Griessen, and B. I. Ivlev, Phys. Rev. Lett. 71, 2319 (1993).

${ }^{7}$ S. H. Brongersma, D. G. Degroot, and R. Griessen, Physica C
235-240, 2589 (1994).

${ }^{8}$ S. H. Brongersma, E. Verweij, N. J. Koeman, D. G. Degroot, R. Griessen, and B. I. Ivlev, Thin Solid Films 228, 201 (1994).

${ }^{9}$ J. Guimpel, F. De la Cruz, J. Murduck, and I. K. Huller, Phys. Rev. B 35, 3655 (1987).

${ }^{10}$ C. Hunnekes, H. G. Bohn, W. Schilling, and H. Schulz, Phys. Rev. Lett. 72, 2271 (1987).

${ }^{11}$ J. D. Jackson, Classical Eletrodynamics (John Wiley \& Sons, New York, 1962), see Sec. 3.11, p. 87.

${ }^{12}$ P. A. Venegas and E. Sardella, Phys. Rev. B 58, 5789 (1998). 